Mehmet Kanoğlu • Yunus A. Çengel İbrahim Dinçer

\title{
Efficiency Evaluation of Energy Systems
}

글 Springer 


\section{Contents}

1 Efficiency, Environment, and Sustainability..................... 1

1.1 Introduction ......................................... 1

1.2 Energy, Exergy, Environment, and Sustainable Development...... 1

1.3 Efficiency and Energy Management........................ 6

2 The First and Second Laws of Thermodynamics.................. 9

2.1 Introduction .......................................... 9

2.2 Energy Change and Energy Transfer $\ldots \ldots \ldots \ldots \ldots \ldots \ldots \ldots \ldots \ldots, \quad 10$

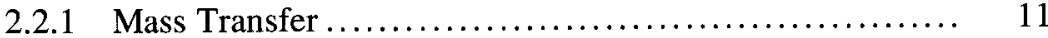

2.2.2 Heat Transfer..................................... 11

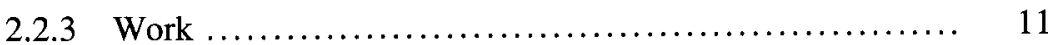

2.3 The First Law of Thermodynamics......................... 12

2.4 The Second Law of Thermodynamics...................... 14

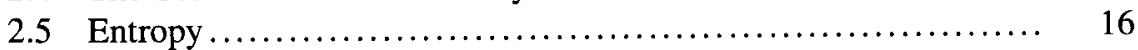

2.5.1 Entropy Balance................................... 18

2.6 Exergy .............................................. 19

2.6.1 What Is Exergy? ............................... 20

2.6.2 Reversibility and Irreversibility ..................... 21

2.6.3 Reversible Work and Exergy Destruction ............... 22

2.6.4 Exergy Change................................... 22

2.6.5 Exergy Transfer Mechanisms........................ 23

2.6.6 Exergy Balance ................................ 24

3 Energy and Exergy Efficiencies............................ 27

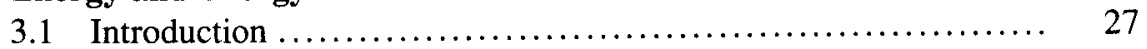

3.2 Efficiencies of Cyclic Devices........................... 30

3.3 Efficiencies of Steady-Flow Devices ....................... 36

3.3.1 Turbine ......................................... 36

3.3.2 Compressor................................... 38 
3.3.3 Pump $\ldots \ldots \ldots \ldots \ldots \ldots \ldots \ldots \ldots \ldots \ldots \ldots \ldots \ldots \ldots \ldots \ldots \ldots \ldots \ldots \ldots, \quad 40$

3.3.4 Nozzle ...................................... 42

3.3.5 Throttling Valve................................... 43

3.3.6 Heat Exchanger ................................ 44

3.3.7 Mixing Chamber ................................. 48

3.3.8 Electric Resistance Heating...................... 49

4 Energy Conversion Efficiencies............................ 55

4.1 Conversion Efficiencies of Common Devices................. 55

4.1.1 Electric Resistance Heater ......................... 55

4.1 .2 Electric Water Heater ............................ 56

4.1.3 Natural Gas Water Heater ........................ 56

4.1.4 Combustion Efficiency........................... 56

4.1.5 Heating Values .................................... 57

$4.1 .6 \quad$ Boiler Efficiency ............................... 57

4.1.7 Generator Efficiency and Overall Efficiency ............. 59

4.1 .8 Lighting Efficacy.................................. 59

4.2 Efficiencies of Mechanical and Electrical Devices............... 60

4.3 Cryogenic Turbine Efficiencies.......................... 62

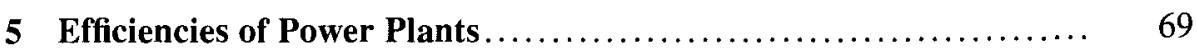

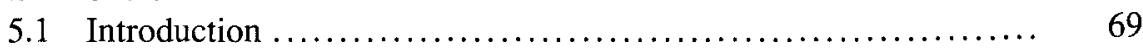

5.2 Efficiencies of Vapor Power Cycles ....................... 70

5.3 Efficiencies of Gas Power Plants ........................... 74

5.4 Efficiencies of Cogeneration Plants......................... 77

5.4.1 Steam-Turbine-Based Cogeneration Plant.............. 79

5.4.2 Gas-Turbine-Based Cogeneration Plant ................. 81

5.5 Efficiencies of Geothermal Power Plants..................... 82

5.6 Energetic and Exergetic Analyses of a Photovoltaic System ...... 89

6 Efficiencies of Refrigeration Systems............................. 95

6.1 Refrigerators and Heat Pumps .............................. 95

6.1.1 Heat Pump Efficiencies ............................... 96

6.1.2 The Carnot Refrigeration Cycle ..................... 98

6.2 Second Law Analysis of Vapor-Compression
Refrigeration Cycle ..................................... 100

6.3 Energy and Exergy Efficiencies of Vapor-Compression Heat Pump Cycle........................................ 106

6.4 Absorption Refrigeration Cycle............................. 109

6.5 Liquefaction of Gases.................................... 111

6.5.1 Linde-Hampson Cycle ............................ 112

6.5.2 Precooled Linde-Hampson Liquefaction Cycle.......... 118 
6.6 Efficiency Analysis of Psychrometric Processes ................ 123

6.6.1 Balance Equations for Common Air-Conditioning Processes...................................... 123

6.6.2 Heating or Cooling ............................... 126

6.6.3 Heating with Humidification...................... 129

6.6.4 Cooling with Dehumidification ...................... 130

6.6.5 Evaporative Cooling ............................ 130

6.6.6 Adiabatic Mixing of Air Streams.................. 131

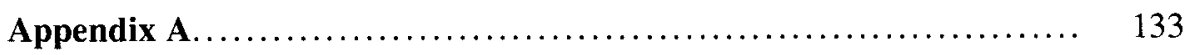

Appendix B........................................ 149

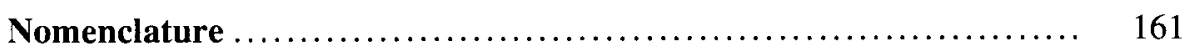

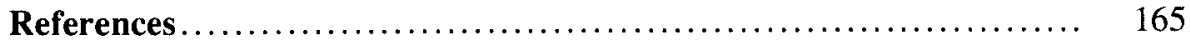

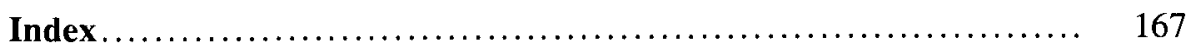

